\title{
Thermal expansion studies on ferroelectric materials
}

\author{
M V RADHIKA RAO and A M UMARJI* \\ Materials Research Centre, Indian Institute of Science, Bangalore 560012, India
}

\begin{abstract}
Thermal expansion data is reported over a wide temperature range $(80-800 \mathrm{~K})$ for $\mathrm{BaTiO}_{3}(\mathrm{BT})$ and $\mathrm{Pb}\left(\mathrm{Fe}_{1 / 2} \mathrm{Nb}_{1 / 2}\right) \mathrm{O}_{3}(\mathrm{PFN})$, belonging to different classes of the ferroelectric materials. The sharp structural phase transitions of $\mathrm{BT}$ result in lowering of the thermal expansion coefficient $(\alpha)$ at the transitions which is proportional to the change in polarization at the transitions. In PFN, a relaxor ferrelectric, lowering of $\alpha$ is spread over a wider temperature range across the dielectric maxima.
\end{abstract}

Keywords. Thermal expansion; ferroelectrics; relaxors; $\mathrm{BaTiO}_{3}: \mathrm{Pb}\left(\mathrm{Fe}_{1: 2} \mathrm{Nb}_{1 / 2}\right) \mathrm{O}_{3}$; electric polarization.

\section{Introduction}

The magnetic and electric contribution to the thermal expansion of solids is well understood through Grüneisen parameter (Krishnan et al 1979). Extensive work on the effect of magnetic transition on thermal expansion has led to the development of invar alloys. The magnetostriction effects result in low thermal expansion coefficient, $\alpha$, in these alloys and, hence, these materials are extensively used in electrical feed-through in ceramic/glass seals. There have not been many reports hitherto describing contribution to the thermal expansion arising from ferro- or antiferroelectric transitions.

$\mathrm{BaTiO}_{3}(\mathrm{BT})$ is a well-known ferroelectric material which exhibits three structural phase transitions (Megaw 1947). The transitions of rhombohedral-to-orthorhombic, orthorhombic-to-tetragonal and tetragonal-to-cubic occur at 183, 273 and $403 \mathrm{~K}$ respectively. These transitions are reflected as sharp changes in the dielectric properties, electric polarization, etc. Early thermal expansion studies of $\mathrm{BaTiO}_{3}$ also show discontinuity in $\Delta L / L$ plots at all the transitions (Kay and Vousden 1949; Rhodes 1951; Shirane and Takeda 1952). However, there is an inconsistency in the reported values of $\alpha$ at different crystal phases and phase transitions. According to Shirane and Takeda (1952), the $\alpha$ values were $5.2,4.6,6.5$ and $9.8 \times 10^{-6} / \mathrm{K}$ in the rhombohedral, orthorhombic, tetragonal and cubic structures respectively. At the rhombohedral-to-tetragonal transition, they have observed an increase in $\alpha$, while at the other two transitions it decreases.

Another class of ferroelectrics, called relaxor ferroelectrics, exhibit a broad dielectric maxima and the temperature of dielectric maximum $\left(T_{\mathrm{m}}\right)$ increases with the increase in measuring frequency. There is no identifiable structural change across the ferroelectriclike transition in these materials. The X-ray patterns of these materials can be indexed on cubic lattice even well below the $T_{\mathrm{m}}$. Some authors have invoked rhombohedral unit cell with rhombohedral angle of $89.92^{\circ}$ for a few materials. However, for all practical purposes, a pseudocubic unit cell is employed (Radhika et al 1996). The reason for the dielectric maxima in relaxor ferroelectrics is thought to be due to entirely a different phenomenon and is yet to be understood completely. A large number of

*Author for correspondence 
lead-based complex oxides having perovskite structure $\left(\mathrm{Pb}\left(B^{\prime} B^{\prime \prime}\right) \mathrm{O}_{3}\right)$ belong to this class of materials. Basically, in such materials, different valent cations occupy the same crystallographic site which builds up charge imbalance, resulting in polar nanodomains. Compositional inhomogeneity, superparaelectric behaviour and growth of polar regions are some of the reasons that have been proposed to explain the relaxor behaviour (Smolenskii 1984; Cross 1994; Tsurumi et al 1994). Generally, it is believed that the polar regions build up much above the $T_{\mathrm{m}}$ and grow with the decrease in temperature. The spontaneous polarization exists even above $T_{\mathfrak{m}}$ and builds up slowly across the broad dielectric maxima region. These relaxor ferroelectrics are known to exhibit low thermal expansion in the region of broad dielectric maxima (Jang et al 1980; Nomura and Uchino 1982). Most of the relaxors have $T_{\mathrm{m}} \mathrm{s}$ below room temperature and the systematic study of thermal expansion, especially much below their $T_{\mathrm{m}}$, has not been reported.

We carried out thermal expansion measurements on normal and relaxor ferroelectrics using LVDT-based push-rod dilatometers developed in our laboratory. The thermal expansion coefficient and the known changes in polarization across the transitions of $\mathrm{BaTiO}_{3}$ were correlated. We have attempted to use this correlation to get information about the nature of transition in the relaxor ferroelectric materials. In order to study the thermal expansion of relaxors, $\mathrm{Pb}\left(\mathrm{Fe}_{1 / 2} \mathrm{Nb}_{1 / 2}\right) \mathrm{O}_{3}(\mathrm{PFN})$ with $T_{\mathrm{m}}$ of $385 \mathrm{~K}$ was selected. Although PFN does not exhibit the frequency dispersion of $T_{\mathrm{m}}$, it shows a diffuse dielectric maxima and can generally be considered as a relaxor ferroelectric (Yokosuka 1993).

\section{Experimental}

$\mathrm{BaTiO}_{3}$ was prepared by the oxalate route (Clabaugh et al 1956). $\mathrm{BaCl}_{2} \cdot 2 \mathrm{H}_{2} \mathrm{O}$ and $\mathrm{TiOCl}_{2}$ were reacted with oxalic acid at $346 \mathrm{~K}$ to get a precipitate of barium titanyl oxalate. The precipitate was then dried and heated at $1173 \mathrm{~K}$ for $7 \mathrm{~h}$. The PVA binder was added to the powder and pelletized. The pellets were sintered at $1623 \mathrm{~K}$ for $4 \mathrm{~h}$.

$\mathrm{Pb}\left(\mathrm{Fe}_{1 / 2} \mathrm{Nb}_{1 / 2}\right) \mathrm{O}_{3}$ was prepared using a Columbite route using respective oxides as the starting materials. $\mathrm{FeNbO}_{4}$ was prepared by mixing the oxides and reacting at $1273 \mathrm{~K}$ for $4 \mathrm{~h}$. It was then mixed with $\mathrm{PbO}$ and reacted at $1173 \mathrm{~K}$ for $1 \mathrm{~h}$. The powder was pelletized and sintered at $1273 \mathrm{~K}$ for $1 \mathrm{~h}$ in lead-rich atmosphere (Radhika et al 1996).

Materials characterization was done by X-ray diffraction and dielectric measurements. Powder X-ray diffraction patterns were recorded on SCINTAG diffractometer at a scan speed of $2^{\circ} / \mathrm{min}$. Dielectric constant and $\tan \delta$ were measured at frequencies from $100 \mathrm{~Hz}$ to $1 \mathrm{MHz}$, using $\mathrm{HP} 4194 \mathrm{~A}$ impedance/gain phase analyzer from room temperature to $473 \mathrm{~K}$.

Thermal expansion measurements were carried out on these samples of length of about $10 \mathrm{~mm}$ from $80 \mathrm{~K}$ to $800 \mathrm{~K}$ on dilatometers developed indigenously (Umarji et al 1997). The high-temperature dilatometer (HTD) can be used from room temperature to $1150 \mathrm{~K}$, whereas, the low-temperature dilatometer (LTD) can be used from $77 \mathrm{~K}$ to $400 \mathrm{~K}$. In both HTD and LTD, change in length of the sample was transferred to the magnetic core of the linear variable differential transformer (LVDT) by the quartz tube. The displacement was measured by monitoring the induced voltage changes in LVDT, which were produced by the movement of the magnetic core. An EG\&G PAR 5210 
lock-in-amplifier (LIA) was used to detect the signal from LVDT. The primary coil was excited by the internal oscillator of the LIA at $1.831 \mathrm{kHz}$ and $0.75 \mathrm{~V}$. The in-phase signal was detected on $1 \mathrm{mV}$ and $3 \mathrm{mV}$ scales for HTD and LTD respectively. Both the dilatometers were calibrated by measuring OFHP copper (SRM-736) and high purity aluminium samples.

The dilatometers working at a heating or cooling rate of $2 \mathrm{~K} / \mathrm{min}$ have a sensitivity of $\Delta L / L=2 \times 10^{-6}$ for sample length of $10 \mathrm{~mm}$ and accuracy of $5 \%$ in the measured thermal expansion coefficients. The output of LIA and the thermocouple output through DMM-196 were acquired by a PC via the IEEE-488 bus. The data was collected for every $90 \mathrm{sec}$ and was averaged for $10 \mathrm{sec}$. The data was then corrected for the contribution from the quartz tubes and the plots of $\Delta L / L$ vs temperature and the derivative curve of $\Delta L / L$, i.e. $\alpha$ (thermal expansion coefficient) vs temperature were plotted.

\section{Results and discussion}

The room temperature X-ray diffraction pattern of BT could be indexed on tetragonal lattice. PFN was completely perovskite with a pseudocubic lattice. BT shows ferroelectric transition $\left(T_{\mathrm{c}}\right)$ at $403 \mathrm{~K}$. The dielectric constant $(\varepsilon)$ and $\tan \delta$ values of BT at room temperature were $2000,0.03$ with a $\varepsilon_{\max }$ of 8000 at $120 \mathrm{~Hz}$. PFN shows a broad dielectric maxima of about 14000 at $100 \mathrm{~Hz}$ at $385 \mathrm{~K}$.

The thermal strain $(\Delta L / L)$ recorded for BT during heating cycle is shown in figure 1a. The strain is calculated with respect to $L$ at room temperature. The exact overlapping of data taken, using HTD and LTD in the temperature range $300-400 \mathrm{~K}$, confirms the reliability of data, calibration of the dilatometers and the analysis procedure adopted. The four structural regions separated by the three transitions are clearly seen as change of slope in $\Delta L / L$ curve. This is more evident in the $\alpha$ vs temperature curve (figure $1 \mathrm{~b}$ ). The average values of $\alpha$ were $3.4,5.85,7.55$ and $12.88 \times 10^{-6} / \mathrm{K}$ in the rhombohedral, orthorhombic, tetragonal and cubic structures respectively. A systematic increase in $\alpha$ with temperature is expected. The temperatures at which $\alpha$ dips agree well with the temperatures of known phase transitions of BT.

The plot of $\Delta L / L$ with temperature recorded, while ramping up the temperature for PFN, is shown in figure 2a. At first glance, the curve appears to be consisting of two parts: a low-thermal expansion and a high-thermal expansion region. The transition from one region to another is gradual. The high temperature $X$-ray diffraction data also indicates a small increase in the cell parameters up to $400 \mathrm{~K}$ and a rapid increase thereafter (Sai Sundar and Umarji 1995). This gradual change in the thermal strain has been observed in $\mathrm{Pb}\left(\mathrm{Mg}_{1 / 3} \mathrm{Nb}_{2 / 3}\right) \mathrm{O}_{3}, \mathrm{~Pb}\left(\mathrm{Zn}_{1 / 3} \mathrm{Nb}_{2 / 3}\right) \mathrm{O}_{3}$ and a solid solution of $\mathrm{Pb}\left(\mathrm{Mg}_{1 / 3} \mathrm{Nb}_{2 / 3}\right) \mathrm{O}_{3}$ and $\mathrm{PbTiO}_{3}$ (Nomura et al 1975; Jang et al 1980).

A careful examination of the curve shows that it has three segments, although not well distinguished. However, this is more evident in the $\alpha$ vs temperature plot shown in figure $2 \mathrm{~b}$. The $\alpha$ slowly increases from $\sim 1 \times 10^{-6} / \mathrm{K}$ to $\sim 2 \times 10^{-6} \mathrm{~K}$ in the temperature region $100-280 \mathrm{~K}$ before lowering to $\sim 0.5 \times 10^{-6} / \mathrm{K}$ in the temperature region of $300-360 \mathrm{~K}$. Then it rapidly increases and saturates above $500 \mathrm{~K}$ to a value of $12 \times 10^{-6} / \mathrm{K}$. It appears that the perovskite lattice has $\alpha$ of $1-3 \times 10^{-6} / \mathrm{K}$ at around $90 \mathrm{~K}$ and steadily reach a value of $\sim 12 \times 10^{-6} / \mathrm{K}$ at about $500 \mathrm{~K}$. Any deviation from this could possibly be attributed to the ferroelectric phase transition. 

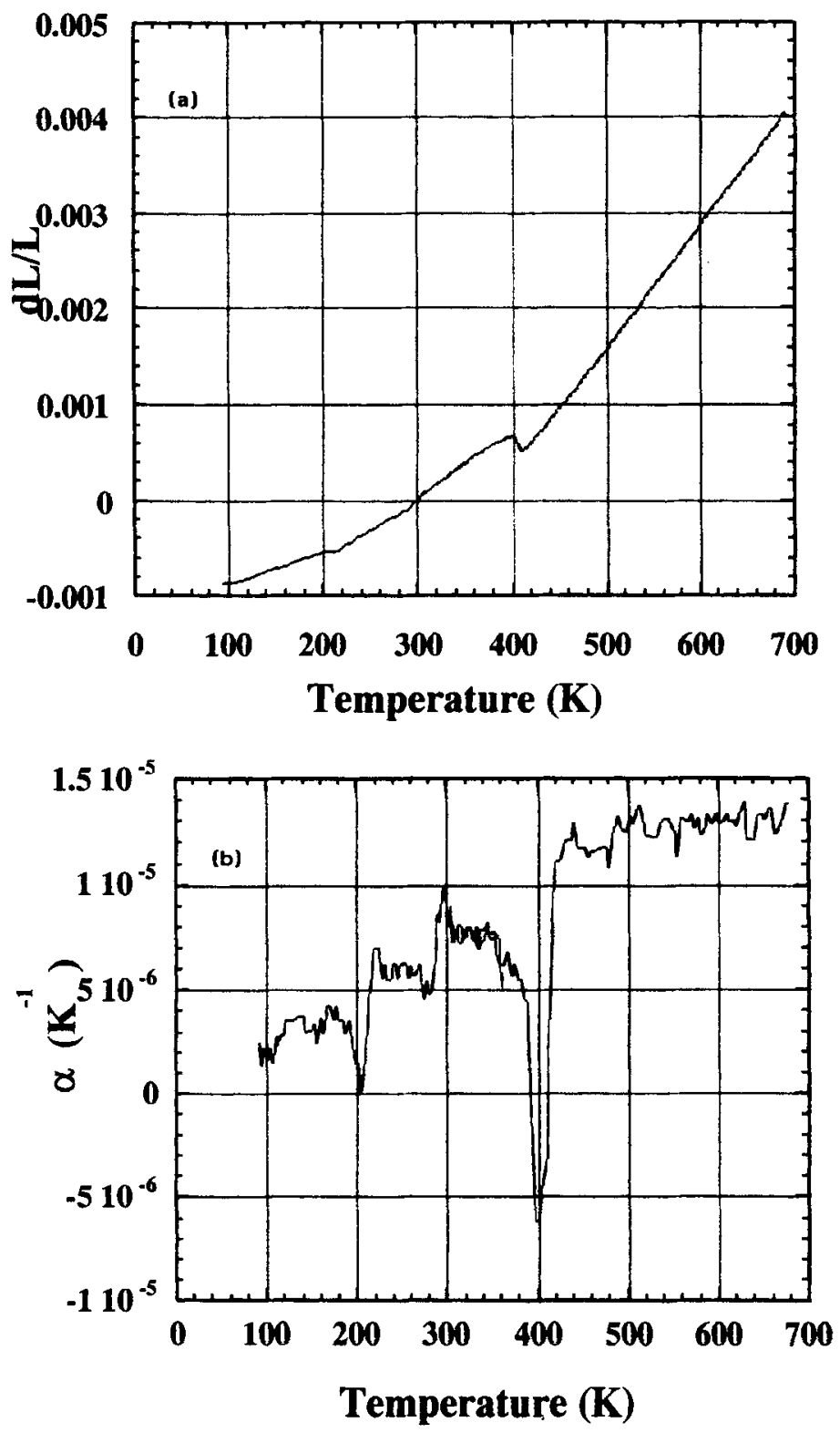

Figure 1. a. Plot of thermal strain, $\Delta L / L$ vs temperature of $\mathrm{BaTiO}_{3}$ and $\mathbf{b}$. plot of $\alpha$, thermal expansion coefficient vs temperature for $\mathrm{BaTiO}_{3}$.

We have attempted to qualitatively relate the changes in $\alpha$ with the phase transitions of BT in order to understand the nature of relaxor ferroelectrics.

In BT, the magnitude of decrease in $\alpha$ appears to be directly related to change in polarization across the phase transitions. The spontaneous polarization changes in single crystal BT are $+9 \cdot 5,+6$ and $-18 \mu \mathrm{C} \mathrm{cm}^{-2}$ at rhombohedral-to-orthorhombic, orthorhombic-to-tetragonal and tetragonal-to-cubic transitions respectively (Jaffe et al 1971). Irrespective of the sign of the change in polarization, $\alpha$ decreases at all 

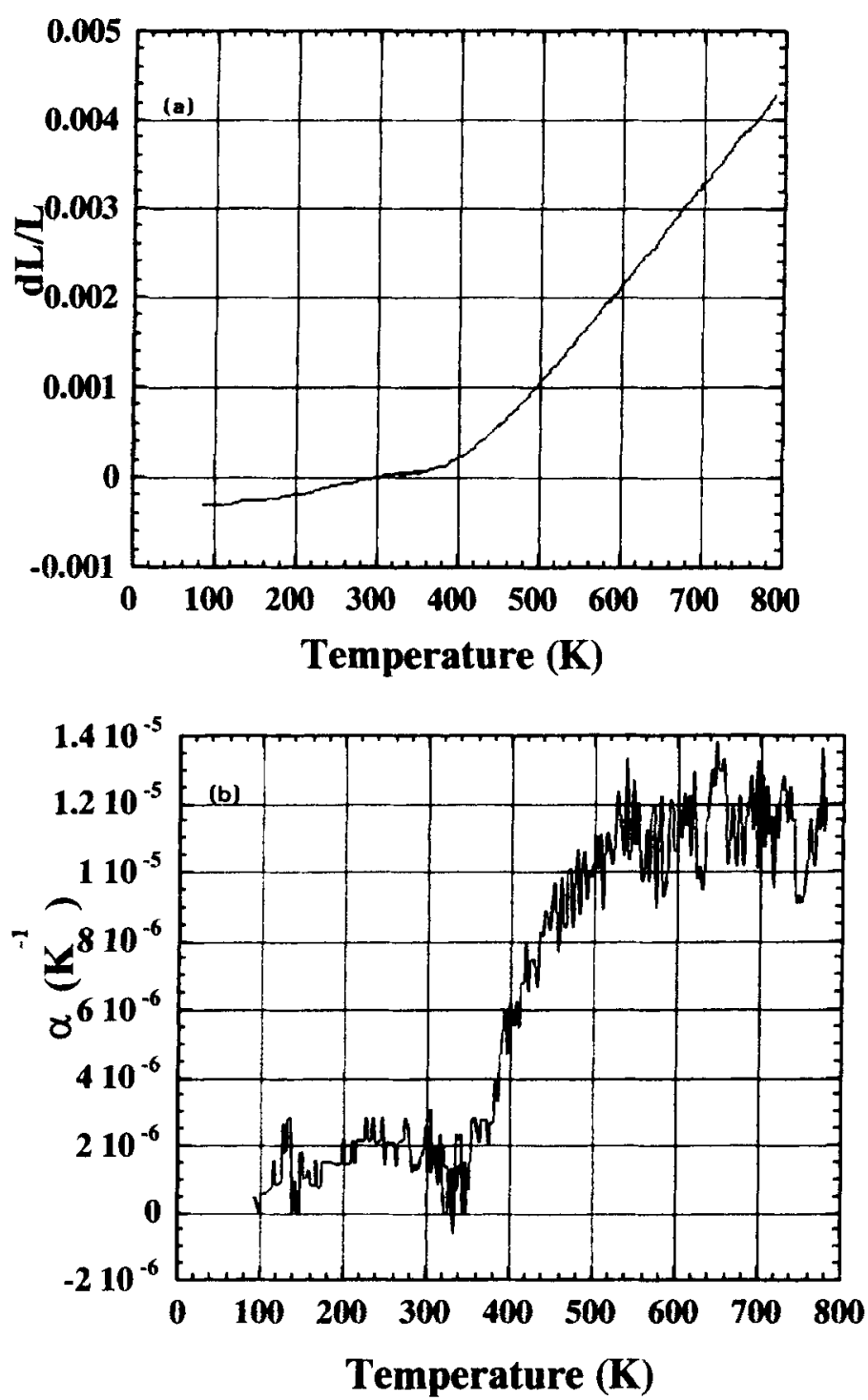

Figure 2. a. Plot of thermal strain, $\Delta L / L$ vs temperature of PFN and b. plot of $\alpha$, thermal expansion coefficient vs temperature for PFN.

the three transitions. This indicates a $P^{2}$ or $|P|$ relation with $\alpha$. During the first order transition, $\alpha$ gets affected over a very narrow temperature range $(10 \mathrm{~K})$ at the transition and the change is sharp.

Assuming a decrease in $\alpha$ to be directly related to changes in the polarization in a material, the behaviour of $\alpha$ of PFN indicates that the polarization changes at much higher temperatures. In case of BT, $\alpha$ remains $\sim 13 \times 10^{-6} / \mathrm{K}$ even about $20 \mathrm{~K}$ higher than $T_{\mathrm{c}}$. In case of PFN, $\alpha$ starts decreasing at about $500 \mathrm{~K}$, i.e. $120 \mathrm{~K}$ above $T_{\mathrm{m}}$. This indicates that the polar regions develop at these temperatures in PFN. Direct evidence of polar regions in $\mathrm{Pb}\left(\mathrm{Mg}_{1 / 3} \mathrm{Nb}_{2 / 3}\right) \mathrm{O}_{3}$ and other relaxor materials has been established 
by TEM and hysteresis loop experiments (Cross 1994). However, the fact that $\alpha$ increases to $\sim 2 \times 10^{-6} / \mathrm{K}$ at lower temperatures $(<300 \mathrm{~K})$, after attaining a low value at $T_{\mathrm{m}}$, could be a suggestion of saturation of polarization. The total temperature region over which $\alpha$ deviates from the perovskite behaviour $(500-300 \mathrm{~K})$ indicates that the polar regions grow in this range, and, hence, a broad dielectric maxima is observed.

\section{Conclusions}

In summary, we have shown that $\alpha$ always shows a decrease at polarization changes in ferroelectric materials. Careful thermal expansion data of relaxor ferroelectric indicate polarization build up over a wide temperature range around $T_{\mathrm{m}}$. Saturation of polarization is speculated at a point below $T_{\mathrm{m}}$. The diffuse nature of ferroelectric phase transition is reflected in broad range of low $\alpha$.

\section{Acknowledgements}

We thank Prof. G Ananthakrishna, MRC, Indian Institute of Science, Bangalore for helpful discussion. We thank the Department of Atomic Energy, Government of India for the financial support and one of the authors (MVRR) thanks CSIR for awarding SRF.

\section{References}

Clabaugh W S, Swiggard E M and Gilchrist R 1956 J. Res. Natl. Bur. Stand. 56289

Cross L E 1994 Ferroelectrics 151305

Jaffe B, Cook W R and Jaffe H 1971 Piezoelectric ceramics (London: Academic Press) p. 53

Jang S J, Uchino K, Nomura S and Cross L E 1980 Ferroelectrics 2731

Kay H F and Vousden P 1949 Philos. Mag. 401019

Krishnan R S, Srinivasan R and Devanarayanan S 1979 Thermal expansion of crystals; International series in: Science of the solid state (Pergamon Press) vol. 12

Megaw H D 1947 Proc. R. Soc. London; Ser. A 189261

Nomura S, Abe M, Kojima F and Uchino K 1975 Jpn. J. Appl. Phys. 141881

Nomura S and Uchino K 1982 Ferroelectrics 41117

Radhika Rao M V, Halliyal A and Umarji A M 1996 J. Am. Ceram. Soc. 79257

Rhodes R G 1951 Acta Crystallogr. 4105

Sai Sundar V V S S and Umarji A M 1995 Mater. Res. Bull. 30427

Shirane G and Takeda A 1952 J. Phys. Soc. Japan 71

Smolenskii G A 1984 Ferroelectrics 53129

Tsurumi T, Soejima K, Kamaya T and Daimon M 1994 Jpn. J. Appl. Phys. 331959

Umarji A M, Senbhagaraman S and Radhika Rao M V 1997 J. Instr. Soc. India 27

Yokosuka M 1993 Jpn. J. Appl. Phys. 321142 